Discrete Comput Geom 34:391-409 (2005)

DOI: $10.1007 / \mathrm{s} 00454-005-1169-\mathrm{z}$

\title{
Sums, Projections, and Sections of Lattice Sets, and the Discrete Covariogram*
}

\author{
Richard J. Gardner, ${ }^{1}$ Paolo Gronchi, ${ }^{2}$ and Chuanming Zong ${ }^{3}$ \\ ${ }^{1}$ Department of Mathematics, Western Washington University, \\ Bellingham, WA 98225-9063, USA \\ Richard.Gardner@wwu.edu \\ ${ }^{2}$ Istituto per le Applicazioni del Calcolo, Sezione di Firenze, \\ Via Madonna del Piano - CNR Edificio F, 50019 Sesto Fiorentino (FI), Italy \\ paolo@fi.iac.cnr.it \\ ${ }^{3}$ School of Mathematics, Peking University, \\ Beijing 100871, People's Republic of China \\ cmzong@math.pku.edu.cn
}

\begin{abstract}
Basic properties of finite subsets of the integer lattice $\mathbb{Z}^{n}$ are investigated from the point of view of geometric tomography. Results obtained concern the Minkowski addition of convex lattice sets and polyominoes, discrete $\mathrm{X}$-rays and the discrete and continuous covariogram, the determination of symmetric convex lattice sets from the cardinality of their projections on hyperplanes, and a discrete version of Meyer's inequality on sections of convex bodies by coordinate hyperplanes.
\end{abstract}

\section{Introduction}

Geometric tomography is the area of mathematics concerning the retrieval of information about an unknown geometric object from data concerning its sections by lines or planes or projections onto lines or planes. When the object is a convex body, many results from convex geometry come into play. Examples are when the data consists of X-rays (Hammer's X-ray problem), or areas of projections (Aleksandrov's projection theorem, Shephard's problem), or areas of central sections (Funk's section theorem, the Busemann-Petty problem). See [14] for more information on all these topics.

* The first author was supported in part by U.S. National Science Foundation Grant DMS-0203527. The third author was supported by the National Science Foundation of China and a special grant from Peking University. 
The unknown object may also be a finite set. Then discrete X-rays measure line counts (see Section 2 for formal definitions), and early work of Rényi on projections of probability distributions and of Ryser on reconstruction of binary matrices from row and column sums can be regarded as contributions to a discrete version of Hammer's X-ray problem; see Note 2.2 of [14]. This topic only took on a life of its own much later, however, with the introduction in 1994 of the term discrete tomography by Shepp. When the unknown finite set is restricted to a lattice, it models the atoms in a crystal; the invention of new methods in electron microscopy that can effectively measure discrete $\mathrm{X}$-rays of crystals was in fact the major motivation behind discrete tomography. Further details can be found in [23] and in the references given below.

Summarizing, we have by now a rich theory of geometric tomography, involving not only X-rays but many other types of data, and a thriving new field called discrete tomography that more or less concerns only discrete X-rays. The purpose of this paper is to lay the groundwork for an extension of discrete tomography that will bring it more in line with geometric tomography. While one can certainly consider arbitrary finite subsets of $\mathbb{R}^{n}$, there seems little doubt that the most interesting problems and applications concern finite subsets of $\mathbb{Z}^{n}$, and we focus on these here.

Corresponding to compact convex sets in $\mathbb{R}^{n}$ are the convex lattice sets in $\mathbb{Z}^{n}$ and an important subclass, the convex polyominoes. Section 3 is a systematic investigation into the behavior of convexity in the lattice setting under Minkowski addition, and it turns out that this fundamental operation is far less predictable than in the continuous framework. For example, the Minkowski sum of convex polyominoes in $\mathbb{Z}^{n}$ is a convex polyomino for $n=2$ but not $n \geq 3$, and while the Minkowski sum of convex lattice sets need not in general be convex, it is true that if $A$ is a convex lattice set in $\mathbb{Z}^{2}$, then $A+A$ and $A+(-A)$ are convex.

In Section 4 we study X-rays and the covariogram. We show that the discrete version of the covariogram is related to the continuous version, and present an example for the discrete covariogram that also provides an example for the continuous covariogram that is in a sense optimal.

Section 5 is motivated by the intriguing possibility, one that apparently has not been considered before, of discrete versions of Aleksandrov's projection theorem: When is an origin-symmetric convex lattice set determined by the cardinalities of its projections on hyperplanes? We establish a connection with the discrete covariogram, and find several examples, one of which shows that a discrete Aleksandrov projection theorem must have additional hypotheses. In Section 6 we turn from projections to sections and find a best-possible discrete version in $\mathbb{Z}^{2}$ of Meyer's inequality on sections of convex bodies by coordinate hyperplanes.

The results of this paper, some positive and some negative, indicate that while the envisioned extension of discrete tomography appears feasible, much more work will have to be done before it reaches the same stage of development as geometric tomography. Several of the basic tools of convex geometry have discrete analogues, but the discrete setting appears to render these far less reliable. Nevertheless, the program seems to us eminently worthwhile. Lattice sets and polyominoes are fundamental objects of interest, not only in mathematics but also in physics and computer science; several remarks indicating connections and applications are scattered throughout this paper. We have also included several fascinating open problems whose solutions will be valuable contributions. 


\section{Definitions and Preliminaries}

As usual, $S^{n-1}$ denotes the unit sphere and $o$ the origin in Euclidean $n$-space $\mathbb{R}^{n}$. If $u \in S^{n-1}$, we denote by $u^{\perp}$ the $(n-1)$-dimensional subspace orthogonal to $u$. The standard orthonormal basis for $\mathbb{R}^{n}$ will be $\left\{e_{1}, \ldots, e_{n}\right\}$.

If $A$ is a set, we denote by $|A|$, int $A$, and conv $A$ the cardinality, interior, and convex hull of $A$, respectively. The dimension of $A$ is the dimension of its affine hull aff $A$, and is denoted by $\operatorname{dim} A$. The notation for the usual orthogonal projection of $A$ on a subspace $S$ is $A \mid S$.

If $A$ and $B$ are subsets of $\mathbb{R}^{n}$, their vector or Minkowski sum is

$$
A+B=\{a+b: a \in A, b \in B\},
$$

and if $r \in \mathbb{R}$, then

$$
r A=\{r a: a \in A\}
$$

Thus $-A$ is the reflection of $A$ in the origin. We also write $D A=A-A=A+(-A)$ for the difference set of $A$.

We write $V_{k}$ for the $k$-dimensional Lebesgue measure in $\mathbb{R}^{n}$, where $k=1, \ldots, n$, and where we identify $V_{k}$ with the $k$-dimensional Hausdorff measure. If $K$ is a $k$-dimensional convex body in $\mathbb{R}^{n}$, then $V(K)$ is its volume $V_{k}(K)$. The notation $d z$ will always mean $d V_{k}(z)$ for the appropriate $k=1, \ldots, n$.

A set is origin symmetric if it is centrally symmetric, with center at the origin.

Let $K$ be a convex body in $\mathbb{R}^{n}$, that is, a compact convex set with nonempty interior. In this case $D K=K-K$ is usually referred to as the difference body of $K$. We denote by

$$
h_{K}(x)=\max \{x \cdot y: y \in K\}
$$

its support function, by

$$
w_{K}(u)=h_{K}(u)+h_{K}(-u),
$$

for $u \in S^{n-1}$, its width function, and by

$$
b_{K}(u)=V\left(K \mid u^{\perp}\right),
$$

for $u \in S^{n-1}$, its brightness function. The projection body of $K$ is the origin-symmetric convex body $\Pi K$ defined by

$$
h_{\Pi K}=b_{K} .
$$

Aleksandrov's projection theorem (see, for example, Theorem 3.3.6 of [14]) states that if $K$ and $L$ are origin-symmetric convex bodies in $\mathbb{R}^{n}$ such that $b_{K}=b_{L}$ (or, equivalently, $\Pi K=\Pi L$ ), then $K=L$. An introduction to the theory of projection bodies is provided in Chapter 4 of [14] and Section 3.5 of [31].

Let $E$ be a bounded Lebesgue measurable subset of $\mathbb{R}^{n}$. The function

$$
g_{E}(x)=V_{n}(E \cap(E+x)),
$$


for $x \in \mathbb{R}^{n}$, is called the covariogram of $E$. Analogously, we define the discrete covariogram of a finite subset $A$ of $\mathbb{R}^{n}$ by

$$
g_{A}(x)=|A \cap(A+x)|,
$$

for $x \in \mathbb{R}^{n}$.

A convex polytope is the convex hull of a finite subset of $\mathbb{R}^{n}$. We sometimes refer to a finite subset of the $n$-dimensional integer lattice $\mathbb{Z}^{n}$ as a lattice set. A convex lattice set is a finite subset of $\mathbb{Z}^{n}$ such that $A=\operatorname{conv} A \cap \mathbb{Z}^{n}$. If $A$ is a convex lattice set, we denote the set $A+A+\cdots+A$ ( $m$ summands) by $A[m]$. (Note that whereas for convex bodies $K$ we have $K+K+\cdots+K=K[m]=m K$, it is not true for finite sets that $A[m]=m A$.)

A polyomino is a finite subset $A$ of $\mathbb{Z}^{n}$ such that the union $A+[0,1]^{n}$ of lattice unit cubes has a connected interior. We also refer to the set $A+[0,1]^{n}$ (itself called a polyomino by many authors) as the animal of the polyomino $A$. A convex polyomino is a polyomino that is also a convex lattice set.

One can also consider weaker forms of convexity. Call a vector $u \in \mathbb{Z}^{n}$ primitive if the line segment $[o, u]$ contains no lattice points other than $o$ and $u$. Then a finite subset $A$ of $\mathbb{Z}^{n}$ is called $u$-convex if the intersection of $A$ with a line $L$ parallel to $u$ consists of consecutive lattice points in $L$. When $n=2$ and $u=e_{1}$ (or $e_{2}$ ), then $u$-convex sets have also been called horizontally convex (or vertically convex, respectively). Such properties and connectivity of finite sets in $\mathbb{Z}^{2}$ were studied by Daurat [10].

Let $A$ be a finite subset of $\mathbb{Z}^{n}$ and let $u \in \mathbb{Z}^{n} \backslash\{o\}$. The discrete $X$-ray of $A$ parallel to $u$ is the function $X_{u} A$ defined by

$$
X_{u} A(v)=\left|A \cap\left(L_{u}+v\right)\right|,
$$

for each $v \in u^{\perp}$, where $L_{u}$ denotes the line through $o$ and $u$. The function $X_{u} A$ is in effect the projection, counted with multiplicity, of $A$ on $u^{\perp}$. For an introduction to the many known results on discrete X-rays and their applications, see [6], [15], [16], and [23].

\section{Minkowski Addition and Convexity}

Certain basic topics such as Helly's theorem have been studied in the context of convex lattice sets (see, for example, [1] and other papers arising from the work of Doignon [13]). There is also a large body of work concerning the lattice-point enumerator, some of which involves obtaining upper or lower bounds for the number of lattice points in the Minkowski sum of two convex polytopes; see, for example, [17], [20], and the references given in these papers. Despite this, we are only aware of one very recent paper, that of Danilov and Koshevoy [9], that considers convexity properties of Minkowski addition in a lattice setting, and this has almost no overlap with our investigation in this section.

When the two convex lattice sets on the left of Fig. 1 are summed by Minkowski addition, the resulting set, on the right of Fig. 1, is not convex. (The authors of [9], who call convex lattice sets "pseudo-convex," were aware of such examples.) Note that these lattice sets are even origin symmetric if placed with their centers at the origin.

Polyominoes are of fundamental interest in many areas, and their convexity properties have also received some attention; see, for example, [12]. The Minkowski sum of two 

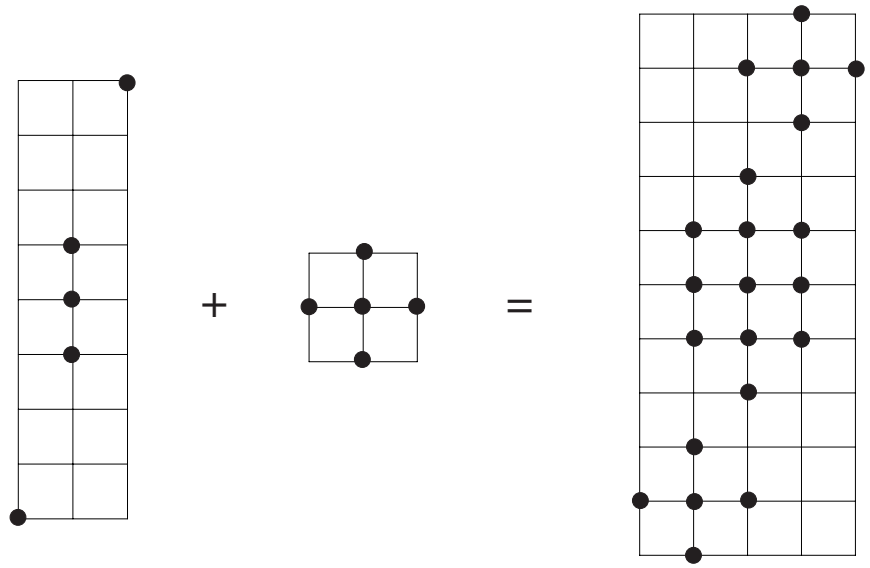

Fig. 1. Minkowski sum does not preserve convexity of lattice sets.

polyominoes is clearly a polyomino, but in three or more dimensions, Minkowski addition of convex lattice sets fails to preserve convexity even for important special cases. For example, let

$$
Q=\operatorname{conv}\{(-1,1,0),(1,-1,0),(1,2,0),(-1,-2,0)\} \cap \mathbb{Z}^{3}
$$

and let

$$
A=\operatorname{conv}\{Q,(0,0,2),(0,0,-2)\} \cap \mathbb{Z}^{3} .
$$

Then $A$ is an origin-symmetric convex polyomino in $\mathbb{Z}^{3}$ consisting of the 11 points in $Q$ and the points $(0,0, \pm 1),(0,0, \pm 2)$. (To see this, note that since $Q$ contains no points with coordinates that are all even, $(0,0,1)$ is the only point in $A$ with the last coordinate 1.) Now clearly $x=(-1,1,2) \in A+A$ and $y=(1,3,0) \in A+A$, but $(x+y) / 2=(0,2,1) \notin A+A$, so $A+A$ is not convex. Moreover, since $-A=A$, $D A=A+(-A)$ is also not convex.

The following construction provides further examples by lifting sets into one dimension higher. Let $A$ and $B$ be convex lattice sets in $\mathbb{Z}^{n}$, and choose origin-symmetric convex polyominoes $C$ and $D$ such that $A \subset C$ and $B \subset D$. In $\mathbb{Z}^{n+1}$ take

$$
X=(\{-1\} \times-A) \cup(\{0\} \times C) \cup(\{1\} \times A)
$$

and

$$
Y=(\{-1\} \times-B) \cup(\{0\} \times D) \cup(\{1\} \times B) .
$$

Then $X$ and $Y$ are convex polyominoes in $\mathbb{Z}^{n+1}$, and the intersection of $X+Y$ with the hyperplane $x_{1}=2$ is precisely $A+B$. If we let $A$ be the origin-symmetric convex polyomino from the previous paragraph, take $B=A$, and apply this construction repeatedly, we obtain origin-symmetric convex polyominoes $X$ in $\mathbb{Z}^{n}, n>3$, such that $X+X=D X$ is not convex. Furthermore, if we apply this construction to the sets $A$ and $B$ in Fig. 1, we obtain origin-symmetric convex polyominoes $X$ and $Y$ in $\mathbb{Z}^{3}$ such that $X+Y$ is not even convex with respect to a coordinate direction. 
In view of these somewhat surprising facts, it is necessary to supply proofs of two theorems supplying positive results in the planar case, that may otherwise seem rather obvious.

Theorem 3.1. If $A$ and $B$ are convex polyominoes in $\mathbb{Z}^{2}$, then $A+B$ is also a convex polyomino.

Proof. Let $A$ and $B$ be convex polyominoes in $\mathbb{Z}^{2}$, and let $P=\operatorname{conv} A$ and $Q=\operatorname{conv} B$. Without loss of generality, suppose that $o$ is a vertex of both $P$ and $Q$. Label the vertices of $P$ clockwise around the boundary by $v_{1}=o, v_{2}, \ldots, v_{m}$, and let $E_{i}=\left[v_{i}, v_{i+1}\right]$, $i=1, \ldots, m$, be the edges of $P$, where the indices are taken modulo $m$. Since $P+Q$ is the union of $P$ and the sets $Q+x$, where $x$ is a boundary point of $P$, it is easy to see that $P+Q=P \cup F \cup G$, where

$$
\begin{gathered}
F=\bigcup_{i=1}^{m}\left(Q+v_{i}\right), \\
G=\bigcup_{i=1}^{m} G_{i},
\end{gathered}
$$

and $G_{i}=E_{i}+\left[o, u_{i}\right]$ is a parallelogram with $u_{i}$ a suitable vertex of $Q$. (Specifically, $u_{i}$ is chosen so that the line parallel to $E_{i}$ through $u_{i}$ supports $Q$ on the same side as the line containing $E_{i}$ supports $P$.) Since $\left(Q+v_{i}\right) \cap \mathbb{Z}^{2} \subset A+B$ for $i=1, \ldots, m$, we have $F \cap \mathbb{Z}^{2} \subset A+B$, so to prove the theorem it will suffice to show that $G \cap \mathbb{Z}^{2} \subset A+B$.

To this end, fix $i \in\{1, \ldots, m\}$. Since $E_{i}$ has endpoints $v_{i}$ and $v_{i+1}$ and $A$ is a polyomino, there is a simple polygonal arc $C_{i}=\bigcup_{j=1}^{p}\left[s_{j}, s_{j+1}\right] \subset P$, where $s_{1}=v_{i}$, $s_{p+1}=v_{i+1}$, each line segment $\left[s_{j}, s_{j+1}\right]$ is of length one, and each $s_{j} \in A$. Moreover, we can ensure that if $R_{i}$ is the open region bounded by $C_{i}$ and $E_{i}$, then $R_{i} \cap \mathbb{Z}^{2}=\emptyset$. Similarly, there is a simple polygonal arc $D_{i}=\bigcup_{k} U_{k} \subset Q$, where each $U_{k}$ is a line segment of length one whose endpoints lie in $B$, such that $D_{i}$ has endpoints $o$ and $u_{i}$ and the open region bounded by $D_{i}$ and $\left[o, u_{i}\right]$ contains no lattice points. Hence, we conclude that

$$
\begin{aligned}
G_{i} \cap \mathbb{Z}^{2} & \subset\left(C_{i}+\left[o, u_{i}\right]\right) \cap \mathbb{Z}^{2}=\bigcup_{j=1}^{p}\left(\left[s_{j}, s_{j+1}\right]+\left[o, u_{i}\right]\right) \cap \mathbb{Z}^{2} \\
& \subset \bigcup_{j=1}^{p}\left(\left[s_{j}, s_{j+1}\right]+D_{i}\right) \cap \mathbb{Z}^{2}=\bigcup_{j=1}^{p+1}\left(s_{j}+D_{i}\right) \cap \mathbb{Z}^{2} \subset A+B .
\end{aligned}
$$

Therefore $G \cap \mathbb{Z}^{2} \subset A+B$, as required.

In Theorem 4 of [28], where planar convex polyominoes are called 4-connected convex digital sets, it is proved that a planar lattice set $A$ is a convex polyomino if and only if it satisfies the strong chord property: Given distinct points $a, b \in A$ and $t \in \mathbb{R}$, there exist distinct points $c, d \in A$ and $r, s \in \mathbb{R}$ such that $c-d$ has length one, $c \in r[-1,1]^{2}+(1-t) a+t b, d \in s[-1,1]^{2}+(1-t) a+t b$, and $r+s<2$. Theorem 3.1 asserts that the strong chord property is preserved by Minkowski addition. 
It is natural to consider a generalization of Theorem 3.1 whereby convexity is replaced by $u$-convexity for a given primitive $u \in \mathbb{Z}^{2}$. However, the resulting statement is false. To see this, let $u=(2,5)$, let $A=[(0,0),(4,0)] \cup[(4,0),(4,8)] \cap \mathbb{Z}^{2}$, and let $B=$ $\{(0,0),(0,1),(0,2)\}$. Then $A$ and $B$ are $u$-convex polyominoes, but $A+B$ is not $u$ convex, since $(0,0) \in A+B$ and $(4,10) \in A+B$ but $(2,5) \notin A+B$.

Despite the previous example, the following version of Theorem 3.1 holds for vertical and horizontal convexity.

Theorem 3.2. If $A$ and $B$ are vertically convex (or horizontally convex) polyominoes in $\mathbb{Z}^{2}$, then $A+B$ is also a vertically convex (or horizontally convex, respectively) polyomino.

Proof. Suppose that $A$ and $B$ are vertically convex polyominoes; the proof for the horizontally convex case is similar. We may assume that $o \in A$ and that $A$ is contained in the closed half-plane lying to the left of the $y$-axis. Suppose that $a_{i} \in A$ and $b_{i} \in B$, $i=1,2$, are such that $a_{1}+b_{1}$ lies below $a_{2}+b_{2}$ on the same vertical line $L$. It suffices to show that each lattice point on $L$ between $a_{1}+b_{1}$ and $a_{2}+b_{2}$ belongs to $A+B$.

From our assumptions it follows that $b_{i}$ lies on or to the right of $L, i=1,2$. Since $A$ is a polyomino, there is a simple polygonal arc $D$ composed of line segments of length one whose endpoints lie in $A$, such that $D$ has endpoints $o$ and $a_{2}$. Moreover, by the vertical convexity of $A$, we may assume that $D$ lies in the closed strip bounded by the vertical lines through $o$ and $a_{2}$. Similarly, there is a simple polygonal arc $E$ composed of line segments of length one whose endpoints lie in $B$, such that $E$ has endpoints $b_{1}$ and $b_{2}$, and by the vertical convexity of $B$, we may assume that $E$ lies in the closed strip bounded by the vertical lines through $b_{1}$ and $b_{2}$.

Without loss of generality, we may assume that the $x$-coordinate of $b_{2}$ is greater than or equal to that of $b_{1}$, so that $a_{2}+b_{1}$ lies on or to the left of $L$. Since the polygonal arc $D+b_{1}$ has endpoints $b_{1}$ and $a_{2}+b_{1}$, it must meet $L$ at some lattice point, $a_{3}+b_{1}$, say. Now for each lattice point $x$ on $E$, all the lattice points on the polygonal arc $D+x$ lie in $A+B$, and $D+x$ meets $L$. It follows that all the lattice points on $L$ between $a_{3}+b_{1}$ and $a_{2}+b_{2}$ belong to $A+B$. Since $A$ is vertically convex, all the lattice points on $L$ between $a_{1}+b_{1}$ and $a_{3}+b_{1}$ also belong to $A+B$. It follows that each lattice point on $L$ between $a_{1}+b_{1}$ and $a_{2}+b_{2}$ belongs to $A+B$.

The next lemma gives a formula for the difference body of a polygon that may be of some independent interest. It will find application in the proof of Theorem 3.4, which, together with the set $A$ defined by (2), shows that it does not hold when $n \geq 3$.

Lemma 3.3. Let $P$ be a convex polygon in $\mathbb{R}^{2}$ with vertices $v_{1}, \ldots, v_{m}$. Then

$$
D P=\left(\bigcup_{i=1}^{m}\left(P-v_{i}\right)\right) \cup\left(\bigcup_{i=1}^{m}\left(-P+v_{i}\right)\right) \text {. }
$$


Proof. By the definition of $D P$, we have

$$
\left(\bigcup_{i=1}^{m}\left(P-v_{i}\right)\right) \cup\left(\bigcup_{i=1}^{m}\left(-P+v_{i}\right)\right) \subset D P,
$$

so it suffices to prove the reverse containment.

Suppose first that $P$ has no pair of parallel edges. Then each oriented edge of $D P$ is either a translate of an oriented edge of $P$, or a translate of an oriented edge of $-P$. (This can be seen by noting that in $\mathbb{R}^{2}$ the surface area measure of $D P$ is the sum of the surface area measures of $P$ and $-P$, and recalling that in $\mathbb{R}^{2}$ the surface area measure of a convex polygon is essentially the set of outer normals to its edges, each weighted by the length of the corresponding edge; see, for example, Section A.1 of [14].) Therefore we can partition $D P$ into triangles as follows:

$$
D P=\bigcup_{i=1}^{2 m} T_{i},
$$

where $T_{i}=\operatorname{conv}\left\{E_{i}, o\right\}$ shares the oriented edge $E_{i}$ with $D P$ and has $o$ as a vertex. Fix $i$ and suppose, without loss of generality, that $E_{i}$ is a translate of an oriented edge $F_{i}$ of $P$. (The argument for the other case is similar.) Let $v \in E_{i}$ have outer unit normal $u$. Then $v \in D P$, so there are $p_{i} \in P, i=1,2$, such that $v=p_{1}-p_{2}$. Since $v$ is in the boundary of $D P, p_{1}$ and $p_{2}$ must be in the boundary of $P$ and moreover have outer unit normals $u$ and $-u$, respectively. The fact that $P$ has no pair of parallel edges implies that $p_{1} \in F_{i}$ and $p_{2}=v_{i}$, say, is the vertex of $P$ opposite to $F_{i}$. As $v \in E_{i}$ was arbitrary, we conclude that $E_{i}=F_{i}-v_{i}$ and therefore $T_{i} \subset P-v_{i}$. In view of (5) we have proved the reverse containment to (4), as required.

Now suppose that $P$ has at least one pair of parallel edges. We can write $P=\lim _{k} P_{k}$, where each $P_{k}$ is a convex polygon with the same number of vertices as $P$ but with no pair of parallel edges. Since (3) holds for each $P_{k}$, it also holds for $P$.

Theorem 3.4. Let $A$ be a convex lattice set in $\mathbb{Z}^{2}$ and let $m \in \mathbb{N}$. If $A[m]=A+$ $A+\cdots+A$ ( $m$ summands $)$ and $D A=A+(-A)$, then $A[m]=(m$ conv $A) \cap \mathbb{Z}^{2}$ and $D A=D(\operatorname{conv} A) \cap \mathbb{Z}^{2}$; in particular, $A[m]$ and $D A$ are also convex lattice sets.

Proof. Let $A$ be a convex lattice set in $\mathbb{Z}^{2}$ and let $m \in \mathbb{N}$. Let $x$ be a vertex of $P=\operatorname{conv} A$, and let $\left\{T_{1}, \ldots, T_{k}\right\}$ be a partition of $P$ into lattice triangles with one vertex at $x$ and the other two at adjacent vertices of $P$. Define $A_{i}=T_{i} \cap \mathbb{Z}^{2}$ and let $y \in m P \cap \mathbb{Z}^{2}$. Then $y \in m T_{i} \cap \mathbb{Z}^{2}$ for some $i \in\{1, \ldots, k\}$, and if $A_{i}[m]=m T_{i} \cap \mathbb{Z}^{2}$, then $y \in A_{i}[m] \subset A[m]$ and $A[m]=m P \cap \mathbb{Z}^{2}$.

Therefore to prove $A[m]=m P \cap \mathbb{Z}^{2}$ we may assume that $P=\operatorname{conv} A$ is a lattice triangle. The proof is by induction on the number $r$ of lattice points in int $P$. Suppose that $r=0$ and without loss of generality let $P=\operatorname{conv}\{o, p, q\}$. Note that $m P$ can be partitioned into translates of $P$ of the form $P+i p+j q, i, j \in\{0, \ldots, m-1\}$, $i+j \leq m-1$, and translates of $-P$ of the form $-P+i p+j q, i, j \in\{0, \ldots, m-1\}$, $1 \leq i+j \leq m$. Let $z \in m P \cap \mathbb{Z}^{2}$. Since int $P$ contains no lattice points, $z$ must actually belong to a translate $P+i p+j q$ of $P$ for some $i, j \in\{0, \ldots, m-1\}, i+j \leq m-1$. Therefore $z \in A+i p+j q \subset A[m]$, and the case $r=0$ is finished. Suppose that the inductive hypothesis holds for $r<s$ and int $P$ contains exactly $s$ lattice points. Let $v$ be 
one of these points, and partition $P$ into three lattice triangles $P_{1}, P_{2}$, and $P_{3}$, each with a vertex at $v$ and two others at vertices of $P$. Let $A_{i}=P_{i} \cap \mathbb{Z}^{2}, i=1,2,3$, and note that $P_{i}=\operatorname{conv} A_{i}$ and int $P_{i}$ has less than $s$ lattice points. Now if $w \in m P \cap \mathbb{Z}^{2}$, then $w \in m P_{i} \cap \mathbb{Z}^{2}$ for some $i$ and so $w \in A_{i}[m] \subset A[m]$. This completes the proof that $A[m]=(m \operatorname{conv} A) \cap \mathbb{Z}^{2}$.

To see that $D A=D(\operatorname{conv} A) \cap \mathbb{Z}^{2}$, let $P=\operatorname{conv} A$ have vertices $v_{1}, \ldots, v_{m}$, and note that $D P=\operatorname{conv} D A$. Let $x \in D P \cap \mathbb{Z}^{2}$. By Lemma 3.3, either $x \in P-v_{i}$ for some $i$ or $x \in-P+v_{i}$ for some $i$. Since $A-v_{i}=\left(P-v_{i}\right) \cap \mathbb{Z}^{2}$ and $-A+v_{i}=\left(-P+v_{i}\right) \cap \mathbb{Z}^{2}$, we have $x \in D A$, as required.

\section{X-Rays, the Covariogram, and Their Discrete Analogs}

Let $E$ be a bounded Lebesgue measurable set in $\mathbb{R}^{n}$. It is a well-known fact that $E$ is determined, up to a set of measure zero, among all bounded Lebesgue measurable sets by its X-rays $X_{u} E$ in all directions $u \in S^{n-1}$; see, for example, Theorem C.1.1 of [14] and the references given there. In particular, if two convex bodies $K$ and $L$ in $\mathbb{R}^{n}$ have equal X-rays in all directions, they must be equal. (For an introduction to X-rays of convex bodies, see Chapter 1 of [14].) The statement that the covariograms of $K$ and $L$ are equal provides weaker information. Indeed, it can be shown that $g_{K}=g_{L}$ if and only if for each $u \in S^{n-1}$, their X-rays $X_{u} K$ and $X_{u} L$ parallel to $u$ are rearrangements of one another. We omit the proof since the main interest here is in finite sets, for which we prove the analogous statement below in Theorem 4.1 .

The covariogram of a bounded Lebesgue measurable set is clearly unchanged by a translation or a reflection in the origin. If $K$ is a convex body in $\mathbb{R}^{n}$, then the support of $g_{K}$ is $D K$. Therefore, if $K$ and $L$ are convex bodies such that $g_{K}=g_{L}$, then $D K=D L$. It follows that a centrally symmetric convex body $K$ is determined up to translation, among all convex bodies, by its covariogram. (As Bianchi [4] remarks, this requires also the observation that $g_{K}(o)=V(K)$ and the fact that in the class of all convex bodies with a given difference body, the unique origin-symmetric member has maximal volume, a consequence of the Brunn-Minkowski inequality.) Matheron [25, p. 86] (or see [18]) observed that $g_{K}=g_{L}$ also implies $\Pi K=\Pi L$.

Let $A$ be a finite subset of $\mathbb{R}^{n}$. Again, $A$ is determined among all finite sets by its discrete $\mathrm{X}$-rays $X_{u} A$ in all directions $u \in S^{n-1}$. Indeed, one can say much more. By a result of Rényi [27], as extended by Heppes [22], if $|A|=m$ then $A$ is determined by any set of $m+1$ discrete X-rays in mutually nonparallel directions.

The discrete covariogram $g_{A}$ of a finite subset $A$ of $\mathbb{R}^{n}$ is also unchanged by a translation or a reflection in the origin, and the support of $g_{A}$ is the difference set $D A=$ $A+(-A)$. By Theorem 3.4, we have for $n=2$ the convenient fact that when $A$ is a convex lattice set, $g_{A}$ is supported by the convex lattice set $D A$. Note that

$$
g_{A}(x)=|\{y \in A: y-x \in A\}|,
$$

the number of chords in $A$ that are translates of the line segment $[o, x]$. Thus the covariogram can be identified with the multiset $A+(-A)$, that is, the set $D A$ where each element is repeated with multiplicity. In particular, $g_{A}=g_{B}$ if and only if $A$ and $B$ have the same set of chords, each repeated with multiplicity, and this is true if and only if the multisets $A+(-A)$ and $B+(-B)$ are equal. 
Finite multisets $A$ and $B$ such that the multisets $A+(-A)$ and $B+(-B)$ are equal are sometimes called homometric. The structure of homometric multisets turns out to be important in X-ray crystallography via the Patterson function, by which the difference set of the atoms in a crystal can be determined from its X-ray diffraction pattern; see, for example, [19]. Rosenblatt and Seymour [29], using algebraic techniques, find an algorithm for reconstructing all multisets $A$ with a given difference multiset $A+(-A)$. The one-dimensional case of this method is applied by Skiena and Sundaram [35] to the so-called partial digest problem in the mapping of DNA.

Let $u \in \mathbb{Z}^{n}$, let $F$ be a finite subset of $\mathbb{Z}^{n}$, and let $j$ be a nonnegative integer. Define

$$
a_{F}(j, u)=\left|\left\{v \in u^{\perp}: X_{u} F(v) \geq j\right\}\right| .
$$

If $A$ and $B$ are convex lattice sets in $\mathbb{Z}^{n}$, we say, by analogy with the usual meaning of the term, that their X-rays $X_{u} A$ and $X_{u} B$ are rearrangements of each other if $a_{A}(j, u)=$ $a_{B}(j, u)$ for each $j$.

Theorem 4.1. Let $A$ and $B$ be convex lattice sets in $\mathbb{Z}^{n}$. Then $g_{A}=g_{B}$ if and only if for each $u \in \mathbb{Z}^{n}$, the $X$-rays $X_{u} A$ and $X_{u} B$ of $A$ and $B$ parallel to $u$ are rearrangements of each other.

Proof. Let $u \in \mathbb{Z}^{n}$ be primitive, let $F$ be a convex lattice set in $\mathbb{Z}^{n}$, and let $j$ be a nonnegative integer. If $v \in u^{\perp}$ and $X_{u} F(v)=k \geq j+1$, then there are exactly $k-j$ chords of $F$ that are translates of $[o, j u]$ lying in the line through $v$ parallel to $u$. Therefore

$$
\begin{aligned}
g_{F}(j u) & =\sum_{k=j+1}^{\infty}(k-j)\left|\left\{v \in u^{\perp}: X_{u} F(v)=k\right\}\right| \\
& =\sum_{k=j+1}^{\infty}(k-j)\left(a_{F}(k, u)-a_{F}(k+1, u)\right) \\
& =\sum_{k=j+1}^{\infty} a_{F}(k, u)
\end{aligned}
$$

for each $j$. (This is a discrete version of the final equation on p. 512 of [18].) The result follows immediately.

The previous theorem shows that, as in the continuous case, the statement that two finite sets have equal discrete covariograms is formally weaker than the statement that all their X-rays are equal. Below, we examine in more detail what information is given by the discrete covariogram. The following connection between continuous and discrete covariograms will be useful.

Lemma 4.2. Let $A$ be a finite subset of $\mathbb{R}^{n}$ with discrete covariogram $g_{A}$, and let $C$ be a bounded Lebesgue measurable set in $\mathbb{R}^{n}$ with continuous covariogram $g_{C}$. Then for all $x \in \mathbb{R}^{n}$,

$$
\sum_{a_{1}, a_{2} \in A} V_{n}\left(\left(C+a_{1}\right) \cap\left(C+a_{2}+x\right)\right)=\sum_{z \in D A} g_{C}(z+x) g_{A}(z) .
$$


Proof. Let $x \in \mathbb{R}^{n}$. We have

$$
\begin{aligned}
\sum_{a_{1}, a_{2} \in A} V_{n}\left(\left(C+a_{1}\right) \cap\left(C+a_{2}+x\right)\right) & =\sum_{a_{1}, a_{2} \in A} V_{n}\left(C \cap\left(C+a_{2}-a_{1}+x\right)\right) \\
& =\sum_{a_{1}, a_{2} \in A} g_{C}\left(a_{1}-a_{2}+x\right) \\
& =\sum_{z \in D A} g_{C}(z+x) g_{A}(z) .
\end{aligned}
$$

Theorem 4.3. Let $A$ and $B$ be finite subsets of $\mathbb{R}^{n}$ with equal discrete covariograms. If $C$ is a bounded Lebesgue measurable set such that

$$
V_{n}(A+C)=|A| V_{n}(C) \quad \text { and } \quad V_{n}(B+C)=|B| V_{n}(C),
$$

then $A+C$ and $B+C$ have equal continuous covariograms.

Proof. The assumption $V_{n}(A+C)=|A| V_{n}(C)$ says that there are no overlaps in the Minkowski sum $A+C$, except for sets of measure zero. Specifically, the assumption implies that for all $a_{1}, a_{2} \in A$ and almost all $c_{1}, c_{2} \in C$, if $a_{1}+c_{1}=a_{2}+c_{2}$, then $a_{1}=a_{2}$ and $c_{1}=c_{2}$. Using this fact, it is easy to see that

$$
V_{n}((A+C) \cap(A+C+x))=\sum_{a_{1}, a_{2} \in A} V_{n}\left(\left(C+a_{1}\right) \cap\left(C+a_{2}+x\right)\right),
$$

for all $x \in \mathbb{R}^{n}$. By Lemma 4.2, we obtain

$$
g_{A+C}(x)=\sum_{z \in D A} g_{C}(z+x) g_{A}(z),
$$

for all $x \in \mathbb{R}^{n}$. The conclusion of the theorem now follows from the hypotheses (7) and $g_{A}=g_{B}$, and the consequence $D A=D B$ of the latter equation.

The following is a result of Cabo and Janssen [8]. Since it appeared only in a technical report, we provide a proof (somewhat shorter than that in [8]).

Proposition 4.4. If $C$ and $D$ are bounded, origin-symmetric, regular (equal to the closure of their interiors), compact subsets of $\mathbb{R}^{n}$ with equal continuous covariograms, then $C=D$.

Proof. It is well known (see, for example, [4]) that for any Lebesgue measurable set $E, g_{E}=1_{E} * 1_{-E}$, the convolution of the characteristic functions of $E$ and $-E$. The origin symmetry implies $1_{C} * 1_{C}=1_{D} * 1_{D}$, and taking Fourier transforms, we obtain $\left(\widehat{1_{C}}\right)^{2}=\left(\widehat{1_{D}}\right)^{2}$. Therefore $\widehat{1_{C}}(x)= \pm \widehat{1_{D}}(x)$, for each $x \in \mathbb{R}^{n}$. Since the Fourier transform is analytic, and any analytic function is determined by its values on a set with a limit point, we conclude that $\widehat{1_{C}}= \pm \widehat{1_{D}}$. Fourier inversion yields $1_{C}=1_{D}$ almost everywhere, and since $C$ and $D$ are regular, we have $C=D$, as required. 
Corollary 4.5. If $A$ and $B$ are finite origin-symmetric subsets of $\mathbb{R}^{n}$ with equal discrete covariograms, then $A=B$.

Proof. Let $C$ be an origin-symmetric $n$-dimensional ball of sufficiently small radius so that (7) holds. Then $g_{A+C}=g_{B+C}$, by Theorem 4.3. Since $A+C$ and $B+C$ are bounded, origin-symmetric, regular compact sets, Proposition 4.4 yields $A+C=B+C$, and it follows that $A=B$.

The covariogram problem, attributed to Matheron (compare p. 86 of [25]) asks whether $g_{K}$ determines a convex body $K$, among all convex bodies, up to translation and reflection in the origin. The answer is affirmative when $n=2$ and $K$ is a polygon (a result of Nagel; see [3] for a complete proof) or when $K$ is $C_{+}^{2}$ (see [4] and [5]), and is negative when $n \geq 4$ (proved by Bianchi [4]). Applications of the covariogram to stereology, image processing, and mathematical morphology are discussed in [7], [30], and [34]. Remarkably, the problem is still open when $n=2$ and 3.

Let $A$ be a finite set in $\mathbb{R}^{n}$. It is natural to ask whether $g_{A}$ determines a convex lattice set $A$, among all convex lattice sets, up to translation and reflection in the origin. The authors of [11] use the algebraic method of Rosenblatt and Seymour [29] to give an algorithm for constructing a finite subset of $\mathbb{Z}^{n}$, if one exists, with a given covariogram. They also note, without giving specific examples, that sets not equal up to translation and reflection in the origin may have the same covariogram. The question raised above is also answered negatively in a strong way by the example depicted in Fig. 2. This displays convex polyominoes $A$ and $B$ that are not congruent, yet satisfy $g_{A}=g_{B}$.

Examples such as these have some relevance to the (continuous) covariogram problem. To see this, let $A$ and $B$ be finite subsets of $\mathbb{Z}^{n}$ such that $g_{A}=g_{B}$. If $C=[0,1]^{n}$, then by Theorem 4.3, the sets $K=A+[0,1]^{n}$ and $L=B+[0,1]^{n}$ have equal covariograms. In particular, if $A$ and $B$ are the convex polyominoes shown in Fig. 2, then the animals $A+[0,1]^{2}$ and $B+[0,1]^{2}$ have equal covariograms.

Since two convex bodies in $\mathbb{R}^{n}$ have equal covariograms if and only if for each $u \in S^{n-1}$, their X-rays parallel to $u$ are rearrangements of one another, this condition is much stronger than the property that the two bodies have equal chord length distributions; indeed, the distributions of lengths of chords parallel to any given direction must be equal. The previous example can therefore be viewed as related to an example of Mallows and Clark [24]. These authors answered an old question of Blaschke by exhibiting two noncongruent convex polygons with equal chord length distributions. Thus the polygons $A+[0,1]^{2}$ and $B+[0,1]^{2}$ have the stronger property that their covariograms are equal,
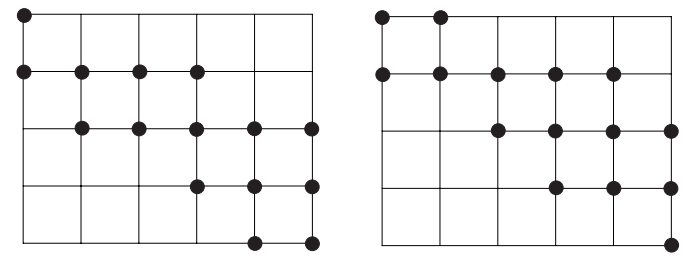

Fig. 2. Noncongruent convex polyominoes with equal covariograms. 
but on the other hand the polygons are not convex. Indeed, they cannot be convex by Nagel's result mentioned above, but they are "close to convex" since they arise from convex polyominoes, and in this sense the example is probably optimal.

\section{Projections of Finite Sets}

To set the scene, we briefly describe some previous work on projections of finite sets. Motivated by a file management problem, Schwenk and Munro [33] consider the minimum of the geometric mean of the cardinality of projections of a finite subset $A$ of $\mathbb{R}^{n}$ onto coordinate subspaces of fixed dimension $k$. Their result in the case $k=n-1$, for example, is that

$$
\left(\prod_{j=1}^{n}|A| e_{j}^{\perp} \mid\right)^{1 / n} \geq|A|^{(n-1) / n} .
$$

However, this follows immediately from (and is equivalent to) the Loomis-Whitney inequality (see, for example, p. 340 of [14]), as can be seen by replacing each point in $A$ by a small cube whose facets are parallel to the coordinate hyperplanes. In [33] a more general inequality is given, involving projections onto all $k$-dimensional coordinate subspaces of $\mathbb{R}^{n}$, but this too follows from a corresponding generalization of the LoomisWhitney inequality due to Hadwiger [21, Theorem 4.4.2]. Schwenk [32] tackles a variant of the problem above in which the geometric mean is replaced by the maximum, and solves this when $n=3$ and $k=2$, noting that the general case appears very difficult.

We begin our investigation with the following connection between projections of convex lattice sets and the discrete covariogram.

Lemma 5.1. If $A$ is a convex lattice set in $\mathbb{Z}^{n}$ and $u \in \mathbb{Z}^{n}$ is primitive, then

$$
|A| u^{\perp}|=| A \mid-g_{A}(u) .
$$

Proof. By (6), we have

$$
|A|-g_{A}(u)=g_{A}(0 u)-g_{A}(1 u)=a_{A}(1, u)=\left|\left\{v \in u^{\perp}: X_{u} A(v) \geq 1\right\}\right|=|A| u^{\perp} \mid .
$$

By analogy with the standard terminology for projection bodies (see, for example, Chapter 4 of [14]), we write $\Pi A=\Pi B$ if

$$
|A| u^{\perp}|=| B\left|u^{\perp}\right|,
$$

for all $u \in \mathbb{Z}^{n}$, and say that $A$ and $B$ have equal projection counts. (Note that $\Pi A$ and $\Pi B$ are not defined separately as sets.)

Corollary 5.2. Let $A$ and $B$ be convex lattice sets in $\mathbb{Z}^{n}$. If $g_{A}=g_{B}$, then $\Pi A=\Pi B$.

Proof. Note that $\Pi A=\Pi B$ if and only if $|A| u^{\perp}|=| B\left|u^{\perp}\right|$ for all primitive $u \in \mathbb{Z}^{n}$. Since $g_{A}(o)=|A|$, the implication $g_{A}=g_{B} \Rightarrow \Pi A=\Pi B$ follows immediately from Lemma 5.1. 

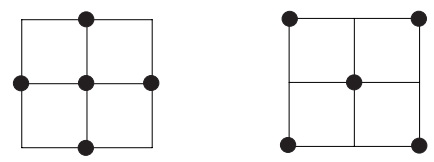

Fig. 3. Nonhomothetic lattice sets with equal projection counts.

By analogy with Aleksandrov's projection theorem, it is natural to ask whether, if centrally symmetric convex lattice sets have equal projection counts, they must be translates of each other. The symmetry assumption is necessary, since it follows from Corollary 5.2 that the convex lattice sets $A_{1}$ and $B_{1}$ shown in Fig. 2 satisfy $\Pi A_{1}=\Pi B_{1}$, yet they are not translates of each other (nor even congruent). Convexity is clearly essential, but one can ask whether arbitrary finite lattice sets with equal projection counts must be homothetic. However, this is false, as the sets $A_{2}$ and $B_{2}$ in Fig. 3 demonstrate.

Surprisingly, there are actually noncongruent origin-symmetric convex lattice sets $A$ and $B$ in $\mathbb{Z}^{2}$ with $\Pi A=\Pi B$, as in Fig. 4 . The two on the left in Fig. 4 are also convex polyominoes; while they are not equal up to a direct rigid motion, each is a reflection of the other in the line $y=x$. The convex lattice set on the right (contributed by a referee) in Fig. 4 is not a convex polyomino.

For any two of the sets in Fig. $4, A_{3}$ and $B_{3}$ say, we have of course $D A_{3} \neq D B_{3}$, since if $D A_{3}=D B_{3}$, the origin symmetry of the sets and Theorem 3.4 would imply that $A_{3}=B_{3}$. With the symmetry condition removed, however, it is possible to find noncongruent convex polyominoes with equal projection counts and equal difference sets. The following general construction can produce such examples. Let $C$ be a finite origin-symmetric set in $\mathbb{R}^{n}$, and let $p \in C$ and $q \in C \backslash\{p,-p\}$ be such that there are points $r \in C \backslash\{p, q\}$ and $s \in C \backslash\{-p, q\}$ such that $r \in[p, q]$ and $s \in[-p, q]$. Let $A=C \backslash\{p, q\}$ and $B=C \backslash\{-p, q\}$. Then $\Pi A=\Pi B$. (Even more general constructions along these lines are possible.)

If we apply this construction with $C=\operatorname{conv}\{p,-p, q,-q\} \cap \mathbb{Z}^{2}$, where $p=(-2,1)$ and $q=(0,-1)$, we obtain the convex polyominoes $A_{4}$ and $B_{4}$ shown in Fig. 5. Note that these satisfy not only $\Pi A_{4}=\Pi B_{4}$, but also $D A_{4}=D B_{4}$. They also show that the converse of Corollary 5.2 is false, since $g_{A_{4}}(x)=2 \neq 1=g_{B_{4}}(x)$ when $x=(-2,2)$.

Now consider the convex polyominoes $A_{5}$ and $B_{5}$ on the left and right of Fig. 6. Here we also have $D A_{5}=D B_{5}$, but since $\left|A_{5}\right| \neq\left|B_{5}\right|$, we have $\Pi A_{5} \neq \Pi B_{5}$ and hence $g_{A_{5}} \neq g_{B_{5}}$.
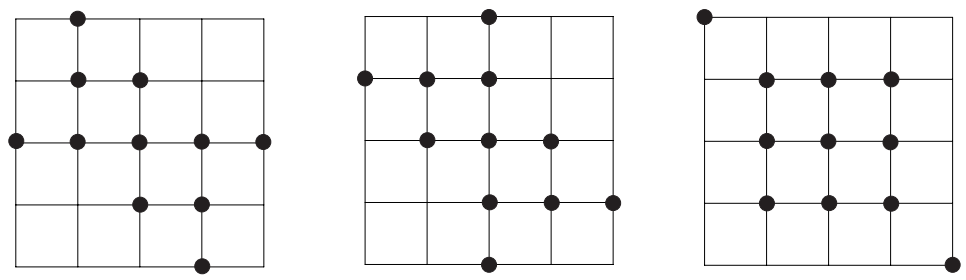

Fig. 4. Noncongruent origin-symmetric convex lattice sets with equal projection counts. 

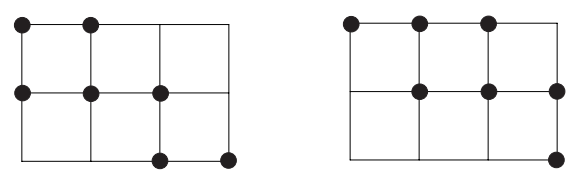

Fig. 5. Noncongruent convex polyominoes with equal projection counts and difference sets.

Despite the example shown in Fig. 3, it might be possible to find a conditional discrete version of Aleksandrov's projection theorem, even in $\mathbb{Z}^{2}$. The following question also remains open, even for origin-symmetric convex polyominoes.

Problem 5.3. Let $n \geq 3$, and let $A$ and $B$ be centrally symmetric convex lattice sets in $\mathbb{Z}^{n}$ with $\operatorname{dim} A=\operatorname{dim} B=n$ such that for each $u \in \mathbb{Z}^{n}$, we have

$$
|A| u^{\perp}|=| B\left|u^{\perp}\right|
$$

Is $A$ a translate of $B$ ?

\section{Sections of Finite Sets}

Dual to Aleksandrov's projection theorem is Funk's section theorem (see Theorem 7.2.6 of [14]), which implies that origin-symmetric convex bodies whose intersections with hyperplanes through the origin have equal volumes must be equal. It is easy to see that the discrete analog of this fact holds. Indeed, a stronger statement is true. Suppose that $A$ and $B$ are origin-symmetric convex lattice sets in $\mathbb{Z}^{n}$ such that for each $u \in \mathbb{Z}^{n}$ we have

$$
\left|A \cap u^{\perp}\right| \leq\left|B \cap u^{\perp}\right|
$$

Let $v \in \mathbb{Z}^{n}$ and let $L_{v}$ be the line through $o$ and $v$. Clearly we can choose $u \in \mathbb{Z}^{n}$ so that $L_{v} \subset u^{\perp}, A \cap u^{\perp}=A \cap L_{v}$, and $B \cap u^{\perp}=B \cap L_{v}$. Then $\left|A \cap L_{v}\right| \leq\left|B \cap L_{v}\right|$. It follows by convexity and origin symmetry that $A \subset B$. In particular, the dual question to Problem 5.3 has an affirmative answer.

Let $A$ be a convex lattice set. For $u \in \mathbb{Z}^{n}$, let

$$
m_{A}(u)=\max \left\{\left|A \cap\left(u^{\perp}+x\right)\right|: x \in \mathbb{Z}^{n}\right\} .
$$

Suppose that $n=2$. If $v \in \mathbb{Z}^{n}$ is primitive and parallel to $u^{\perp}$, then $k v \in D A$ if and only if there are $x, y \in A$ with $x-y=k v$ and hence if and only if $k \leq m_{A}(u)-1$. It follows that convex lattice sets $A$ and $B$ in $\mathbb{Z}^{2}$ satisfy $m_{A}=m_{B}$ if and only if $D A=D B$. The noncongruent convex polyominoes shown in Fig. 6 therefore satisfy $m_{A}=m_{B}$.
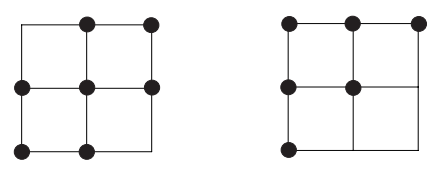

Fig. 6. Noncongruent convex polyominoes with equal difference sets but different cardinalities. 
However, the following is a discrete version of the old and still-unsolved problem of Bonnesen (see Problem 8.10(i) of [14]).

Problem 6.1. Let $A$ and $B$ be convex lattice sets in $\mathbb{Z}^{n}$ with $\operatorname{dim} A=\operatorname{dim} B=n$. If $n \geq 3$ and $m_{A}=m_{B}$, is $A=B$ up to translation and reflection in the origin?

For the remainder of this section, we consider a discrete version of the following dual Loomis-Whitney inequality proved by Meyer [26]. This states that if $K$ is a convex body in $\mathbb{R}^{n}$, then

$$
V(K)^{n-1} \geq d_{n} \prod_{i=1}^{n} V\left(K \cap e_{i}^{\perp}\right)
$$

where

$$
d_{n}=\frac{((n-1) !)^{n}}{(n !)^{n-1}},
$$

and equality holds if and only if $K$ is a cross-polytope with vertices on the coordinate axes.

Problem 6.2. Find the best-possible constants $c_{n}$ such that if $A$ is a convex lattice set in $\mathbb{Z}^{n}$, then

$$
|A|^{n-1} \geq c_{n} \prod_{i=1}^{n}\left|A \cap e_{i}^{\perp}\right| .
$$

We claim that $c_{n} \leq d_{n}$, where $d_{n}$ is given by (10). To see this, one can use the fact that for each convex body $K$ in $\mathbb{R}^{n}$, the number $G(K)$ of lattice points in $K$ satisfies

$$
|G(m K)-V(m K)|=O\left(m^{n-1}\right),
$$

for large $m$; see, for example, [2]. Now let $K=\operatorname{conv}\left\{ \pm e_{i}, i=1, \ldots, n\right\}$ be the standard cross-polytope in $\mathbb{R}^{n}$, and let $\varepsilon>0$ be given. Let $m \in \mathbb{N}$ and $A_{m}=m K \cap \mathbb{Z}^{n}$. Then, using (12) and the equality condition for (9), we have

$$
\begin{aligned}
\left|A_{m}\right|^{n-1} \geq\left(V(m K)-O\left(m^{n-1}\right)\right)^{n-1} & =V(m K)^{n-1}-O\left(m^{n^{2}-n-1}\right) \\
& =d_{n} \prod_{i=1}^{n} V\left((m K) \cap e_{i}^{\perp}\right)-O\left(m^{n^{2}-n-1}\right) \\
& \geq d_{n} \prod_{i=1}^{n}\left(\left|A_{m} \cap e_{i}^{\perp}\right|-O\left(m^{n-2}\right)\right)-O\left(m^{n^{2}-n-1}\right) \\
& =d_{n} \prod_{i=1}^{n}\left|A_{m} \cap e_{i}^{\perp}\right|-O\left(m^{n^{2}-n-1}\right) \\
& \geq\left(d_{n}-\varepsilon\right) \prod_{i=1}^{n}\left|A_{m} \cap e_{i}^{\perp}\right|
\end{aligned}
$$

for sufficiently large $m$. This proves the claim. 
Let $A=\left\{ \pm e_{i}: 1 \leq i \leq n-1, \pm j e_{n}: 0 \leq j \leq m\right\} \subset \mathbb{Z}^{n}$. Then $|A|=2 m+2 n-1$, $\left|A \cap e_{n}^{\perp}\right|=2 n-1$, and $\left|A \cap e_{i}^{\perp}\right|=2 m+2 n-3$. This shows that $c_{n} \leq 1 /(2 n-1)$. Note that $d_{3}=\frac{2}{9}>\frac{1}{5}$, while $d_{n}<1 /(2 n-1)$ for $n \geq 4$.

Theorem 6.3. If $A$ is a convex lattice set in $\mathbb{Z}^{2}$, then

$$
|A| \geq \frac{1}{3} \prod_{i=1}^{2}\left|A \cap e_{i}^{\perp}\right|
$$

and the constant $\frac{1}{3}$ is the best possible.

Proof. It will be convenient to set $x=\left|A \cap e_{1}^{\perp}\right|$ and $y=\left|A \cap e_{2}^{\perp}\right|$.

Suppose first that $o \in A$. Let $A \cap e_{1}^{\perp}=\left\{-a_{2},-a_{2}+1, \ldots, a_{1}\right\}$ and $A \cap e_{2}^{\perp}=$ $\left\{-b_{2},-b_{2}+1, \ldots, b_{1}\right\}$. If $a_{2}=b_{2}=0$, we have

$$
|A| \geq\left(a_{1}+1\right)\left(b_{1}+1\right) / 2+1 .
$$

Using (14) in each quadrant, we see that in general we have

$$
\begin{aligned}
|A| \geq & \left(\frac{\left(a_{1}+1\right)\left(b_{1}+1\right)+\left(a_{1}+1\right)\left(b_{2}+1\right)+\left(a_{2}+1\right)\left(b_{1}+1\right)+\left(a_{2}+1\right)\left(b_{2}+1\right)}{2}\right) \\
& +4-\left(a_{1}+a_{2}+1\right)-\left(b_{1}+b_{2}+1\right)-1 \\
= & \frac{\left(a_{1}+a_{2}\right)\left(b_{1}+b_{2}\right)}{2}+3=\frac{(x-1)(y-1)}{2}+3 .
\end{aligned}
$$

Now if $x, y \geq 3$, we have

$$
\frac{(x-1)(y-1)}{2}+3 \geq \frac{x y}{3} \quad \Leftrightarrow \quad \frac{(x-3)(y-3)}{6}+2 \geq 0,
$$

which is true. If $x \leq 2$, say, then $|A| \geq y+1 \geq(x y) / 2+1 \geq(x y) / 3$.

Now suppose that $o \notin A$. Since $A$ is convex, it suffices to consider the situation when $A \cap e_{1}^{\perp}=\left\{a_{2}, a_{2}+1, \ldots, a_{1}\right\}$ and $A \cap e_{2}^{\perp}=\left\{b_{2}, b_{2}+1, \ldots, b_{1}\right\}$, where $0<a_{2} \leq a_{1}$ and $0<b_{2} \leq b_{1}$. Using (14) again, we obtain

$$
\begin{aligned}
|A| & \geq \frac{\left(a_{1}+1\right)\left(b_{1}+1\right)}{2}+1-\left(\frac{\left(a_{2}+1\right)\left(b_{2}+1\right)}{2}-1\right) \\
& =\frac{\left(a_{1}-a_{2}+1\right)\left(b_{1}-b_{2}+1\right)+a_{2}\left(b_{1}-b_{2}\right)+b_{2}\left(a_{1}-a_{2}\right)}{2} \geq \frac{x y}{2},
\end{aligned}
$$

an even better estimate in this case than the one required.

We have proved that $c_{2} \geq \frac{1}{3}$. Let $A=\{( \pm 1,0),(0, \pm i): 0 \leq i \leq m\}$. Then $|A|=$ $2 m+3, x=3$, and $y=2 m+1$, which shows that $c_{2} \leq \frac{1}{3}$.

\section{Acknowledgment}

We thank the referees for helpful suggestions and contributions, including two references and the picture at the right of Fig. 4, that improved the paper. 


\section{References}

1. I. Bárány and J. Matoušek, A fractional Helly theorem for convex lattice sets, Adv. Math. 174 (2003), 227-235.

2. U. Betke and K. Böröczky, Jr., Asymptotic formulae for the lattice point enumerator, Canad. J. Math. 51 (1999), 225-249.

3. G. Bianchi, Determining convex polygons from their covariograms, Adv. in Appl. Prob. 34 (2002), 261266.

4. G. Bianchi, Matheron's conjecture for the covariogram problem, J. London Math. Soc. (2) 71 (2005), 203-220.

5. G. Bianchi, F. Segala, and A. Volčič, The solution of the covariogram problem for plane $C_{+}^{2}$ bodies, J. Differential Geom. 60 (2002), 177-198.

6. S. Brunetti and A. Daurat, An algorithm reconstructing lattice convex sets, Theoret. Comput. Sci. 304 (2003), 35-57.

7. A. Cabo and A. Baddeley, Estimation of mean particle volume using the set covariance function, $A d v$. in Appl. Probab. 35 (2003), 27-46.

8. A. Cabo and R. H. P. Janssen, Cross-variance functions characterise bounded closed regular sets, CWI Technical Report, Amsterdam, 1994.

9. V. I. Danilov and G. A. Koshevoy, Discrete convexity and unimodularity—I, Adv. Math. 189 (2004), 301-324.

10. A. Daurat, Connexité et convexité directionelle dans $\mathbb{Z}^{2}$, in: $C N R$ 'IUT2000, vol. 1, Presses Universitaires d'Orléans, Orléans, 2000, pp. 341-350.

11. A. Daurat, Y. Gérard, and M. Nivat, The chords' problem, Theoret. Comput. Sci. 282 (2002), 319-336.

12. I. Debled-Rennesson, J.-L. Rémy, and J. Rouyer-Degli, Detection of the discrete convexity of polyominoes, Discrete Appl. Math. 125 (2003), 115-133.

13. J.-P. Doignon, Convexity in crystollographical lattices, J. Geom. 3 (1973), 71-85.

14. R. J. Gardner, Geometric Tomography, Cambridge University Press, New York, 1995. Corrections and update available at http://www. ac. wwu. edu/ gardner.

15. R. J. Gardner and P. Gritzmann, Discrete tomography: determination of finite sets by X-rays, Trans. Amer. Math. Soc. 349 (1997), 2271-2295.

16. R. J. Gardner and P. Gritzmann, Uniqueness and complexity in discrete tomography, in: Discrete Tomography: Foundations, Algorithms and Application, ed. by G. T. Herman and A. Kuba, Birkhäuser, Boston, MA, 1999, pp. 85-113.

17. R. J. Gardner and P. Gronchi, A Brunn-Minkowski inequality for the integer lattice, Trans. Amer. Math. Soc. 353 (2001), 3995-4024.

18. R. J. Gardner and G. Zhang, Affine inequalities and radial mean bodies, Amer. J. Math. 120 (1998), 493-504.

19. J. P. Glusker, B. K. Patterson, and M. Rossi, Patterson and Pattersons, Oxford University Press, New York, 1987.

20. P. Gritzmann and J. M. Wills, Lattice points, in: Handbook of Convexity, ed. by P. M. Gruber and J. M. Wills, North-Holland, Amsterdam, 1993, pp. 765-797.

21. H. Hadwiger, Vorlesungen über Inhalt, Oberfläche und Isoperimetrie, Springer, Berlin, 1957.

22. A. Heppes, On the determination of probability distributions of more dimensions by their projections, Acta Math. Acad. Sci. Hung. 6 (1956), 403-410.

23. G. T. Herman and A. Kuba, Discrete Tomography: Foundations, Algorithms, and Applications, Birkhäuser, Boston, MA, 1999.

24. C. L. Mallows and J. M. C. Clark, Line-intercept distributions do not characterize plane sets, J. Appl. Probab. 7 (1970), 240-244.

25. G. Matheron, Random Sets and Integral Geometry, Wiley, New York, 1975.

26. M. Meyer, A volume inequality concerning sections of convex sets, Bull. London Math. Soc. 20 (1988), $151-155$.

27. A. Rényi, On projections of probability distributions, Acta Math. Acad. Sci. Hung. 3 (1952), 131-142.

28. C. Ronse, A strong chord property for 4-connected convex digital sets, Comput. Vision Graphics Image Process. 35 (1986), 259-269.

29. J. Rosenblatt and P. D. Seymour, The structure of homometric sets, SIAM J. Algebraic Discrete Methods 3 (1982), 343-350. 
30. M. Schmitt, On two inverse problems in mathematical morphology, in: Mathematical Morphology in Image Processing, ed. by E. R. Dougherty, Marcel Dekker, New York, pp. 151-169.

31. R. Schneider, Convex Bodies: The Brunn-Minkowski Theory, Cambridge University Press, Cambridge, 1993.

32. A. J. Schwenk, How to minimize the largest shadow of a finite set, in: Graphs and Applications, Boulder, CO, 1982, Wiley Interscience, New York, 1985, pp. 279-294.

33. A. J. Schwenk and J. I. Munro, How small can the mean shadow of a set be?, Amer. Math. Monthly 90 (1983), 325-329.

34. J. Serra, Image Analysis and Mathematical Morphology, Academic Press, London, 1982.

35. S. S. Skiena and G. Sundaram, A partial digest approach to restriction site mapping, Bull. Math. Biol. 56 (1994), 275-294.

Received August 20, 2004, and in revised form November 29, 2004. Online publication May 12, 2005. 